\title{
Tendência Genética dos Efeitos Direto e Materno sobre os Pesos à Desmama e Pós- Desmama de Bovinos da Raça Tabapuã no Brasil
}

\section{Paulo Bahiense Ferraz Filho ${ }^{1}$, Alcides de Amorim Ramos ${ }^{2}$, Luiz Otávio Campos da Silva ${ }^{3}$, Júlio César de Souza 4 , Maurício Mello de Alencar ${ }^{5}$, Carlos Henrique Mendes Malhado ${ }^{6}$}

RESUMO - Os dados são relativos aos pesos de animais da raça Tabapuã, nascidos no período de 1959 a 1996 em várias regiões brasileiras. As observações foram analisadas com o objetivo de avaliar as mudanças genéticas aditivas diretas e maternas dos pesos padronizados para 205 (P205), 365 (P365) e 550 (P550) dias de idade. As estimativas dos componentes de (co) variância utilizadas no cálculo dos valores genéticos foram obtidas pelo método de máxima verossimilhança restrita livre de derivadas (REML), usando o aplicativo MTDFREML, cujo modelo continha os efeitos aleatórios aditivo direto, materno e de ambiente permanente, além dos efeitos fixos de grupo de contemporâneos (unidade da federação, fazenda, sexo, estação e ano de nascimento do animal) e a covariável idade da vaca ao parto (efeitos linear e quadrático). As tendências genéticas dos efeitos genéticos direto e materno foram estimadas pela regressão, ponderada, das médias anuais dos valores genéticos dos animais. As tendências genéticas dos efeitos direto no período estudado foram 0,134; 0,207, e 0,276 kg/ano, para P205, P365 e P550, respectivamente. Ainda para os três pesos, na mesma ordem, as estimativas das tendências genéticas maternas foram 0,$019 ;-0,011 ; \mathrm{e}-0,022 \mathrm{~kg} /$ ano. Em virtude da variação genética existente, os resultados observados estão bem aquém das mudanças possíveis.

Palavras-chave: gado de corte, herdabilidade, mudança genética

\section{Genetic Trends of the Direct and Maternal Effects for Weaning and Post-Weaning Weights of Tabapuã Cattle in Brazil}

\begin{abstract}
Data related to weights of animals of the Tabapuã breed, born from 1959 to 1996, in several areas of Brazil, were analyzed with the objective of evaluating the direct and maternal genetic trends, for body weights adjusted for 205 (W205), 365 (W365) and 550 (W550) days of age. The (co)variance component estimates used in the calculation of the breeding values were obtained by the restricted maximum likelihood method (REML), with a model containing the random additive direct, maternal and permanent environmental effects, and the fixed effects of contemporary group (unit of the federation, farm, sex, season and year of birth of the animal) and the covariable age of the cow at calving (linear and quadratic effects). The genetic trends of the direct and maternal genetic effects were estimated by the regression of the breeding value annual means on year of birth of the animals. The genetic trends of the direct effects were $0.134,0.199$ and $0.276 \mathrm{~kg} /$ year, for W205, W365 and W550, respectively. The estimates of the maternal genetic trends were, in the same order, $0.019,-0.010$ and $-0.022 \mathrm{~kg} /$ year, respectively. Due to the existing genetic variability on the traits, the genetic changes attained are bellow the possible ones.
\end{abstract}

Key Words: beef cattle, heritability, genetic change

\section{Introdução}

A pecuária bovina de corte brasileira está passando por um processo de modernização, em conseqüência da necessidade de aumentar a eficiência produtiva, causada, em parte, pela abertura de mercados e pela competição por outros tipos de carne. Para manter a competitividade, é preciso aumentar a produtividade dos bovinos, o que pode ser atingido pela combinação de melhores manejos, nutrição, reprodução e sanidade, aliados a animais de maior potencial genético.

Entre os métodos de melhoramento disponíveis para modificar o potencial genético dos animais, a seleção é aquele que, por meio da escolha dos pais que irão produzir a próxima geração, procura aumentar a freqüência dos genes desejáveis na população. Todo programa de seleção deve ser acompanhado

\footnotetext{
${ }^{1}$ Prof. Adjunto da UFMS/DCN. Av. Ranulpho Marques Leal, 3484 - CEP 79610-000 Caixa Postal 210 - Três Lagoas - MS. Bolsista da CAPES E.mail: pbferraz@ceul.ufms.br

2 Prof. Titular da UNESP/DPEA/FCA. Bolsista do CNPq. E.mail: aaramos@fca.umesp.br

3 Pesquisador da Embrapa Gado de Corte. E.mail: locs@cnpgc.embrapa.br

4 Professor Adjunto da UFPR/DZO. E.mail: jcs@ufpr.br

${ }^{5}$ Pesquisador da Embrapa Pecuária Sudeste - bolsista do CNPq. E.mail: mauricio@cppse.embrapa.br

${ }^{6}$ Pós-Graduando da UFC. E.mail: chmmalhado@hotmail.com
} 
periodicamente para que se possa corrigir rumos de forma rápida. Uma das maneiras de se promover o monitoramento dos resultados é por meio da avaliação da mudança genética ao longo do tempo. Desta forma, com o objetivo não só de avaliar o progresso genético que vem sendo alcançado, mas, principalmente, para que os resultados sirvam de elementos orientadores de ações futuras, torna-se de grande importância avaliar a tendência genética ao longo do tempo (Euclides Filho et al., 1997 b).

Características de crescimento, como o peso corporal, medidas na fase inicial do desenvolvimento do animal, são importantes na determinação da eficiência econômica de qualquer sistema de produção de bovinos e podem ser recomendadas como critérios de seleção. No Brasil, os pesos à desmama, ao ano e ao sobreano são características normalmente utilizadas nos programas de avaliação genética de reprodutores (Brasil, 1999), em virtude de suas importâncias para a eficiência dos setores produtivos. É necessário, portanto, que as mudanças genéticas observadas nessas características sejam avaliadas nos rebanhos brasileiros.

O presente trabalho teve como objetivo verificar as tendências genéticas aditivas direta e materna dos pesos à desmama, ao ano e ao sobreano de bovinos da raça Tabapuã, criados em pastos de diferentes regiões do Brasil.

\section{Material e Métodos}

Os dados analisados são provenientes de rebanhos bovinos da raça Tabapuã incluídos no Controle de Desenvolvimento Ponderal da Associação Brasileira de Criadores de Zebu (ABCZ). As características estudadas foram os pesos à desmama, aos $12 \mathrm{e}$ aos 18 meses de idade padronizados, por interpolação, para 205 (P205), 365 (P365) e 550 (P550) dias de idade, respectivamente. Foram considerados apenas os animais criados em regime exclusivo de pastagens, nascidos no período de 1959 a 1996. Em função do pequeno número de informações, os animais nascidos de 1959 a 1968 foram agrupados em um único ano de nascimento (1968) e aqueles nascidos em 1996 foram considerados como nascidos em 1995, pelo mesmo motivo. Observações de animais com peso aos 205, 365 e 550 dias inferiores a 112, 139 e $155 \mathrm{~kg}$ e superiores a 228,315 e $431 \mathrm{~kg}$, respectivamente, foram descartadas.
Para avaliar as tendências genéticas utilizou-se a regressão, ponderada, das médias anuais dos valores genéticos (aditivo, materno e total maternal) sobre o ano de nascimento do animal.

Para obter as estimativas dos componentes de (co)variância e predição dos valores genéticos, empregou-se a metodologia da máxima verossimilhança restrita livre de derivadas, usando um modelo animal e o programa MTDFREML (Boldman et al., 1993). O modelo estatístico considerou os efeitos fixos de grupo de contemporâneos (fazenda, unidade da federação, sexo, estação e ano de nascimento do animal), idade da vaca como covariável (efeitos linear e quadrático) e os efeitos aleatórios genético direto, genético materno e de ambiente permanente. $\mathrm{Na}$ forma matricial o modelo foi:

$$
Y=X b+Z a+M m+W p m+e
$$

em que Y é o vetor das variáveis dependentes (P205, P365 e P550); $b$, o vetor de efeitos fixos no modelo, associados com a matriz de incidência $\mathrm{X} ; a$, o vetor dos efeitos aleatórios de valor genético aditivo direto do animal, associado com a matriz de incidência Z; $m$, o vetor dos efeitos genéticos maternos, associado com a matriz de incidência $\mathrm{M} ; \mathrm{pm}$, o vetor dos efeitos aleatórios de ambiente permanente da vaca, associado com a matriz de incidência $\mathrm{W}$; $e$, o vetor dos efeitos residuais.

Os efeitos genéticos direto e materno são assumidos como correlacionados entre si, enquanto que os de ambiente permanente e residuais não são correlacionados entre si e nem com os efeitos genéticos. A estrutura básica da matriz de variância e covariância para as análises foi, então, descrita como:

$$
v\left[\begin{array}{l}
a \\
m \\
p m \\
e
\end{array}\right]=\left[\begin{array}{cccc}
A \sigma_{a}^{2} & A \sigma_{a m} & 0 & 0 \\
A \sigma_{a m} & A \sigma_{m}^{2} & 0 & 0 \\
0 & 0 & I \sigma_{p m}^{2} & 0 \\
0 & 0 & 0 & I \sigma_{e}^{2}
\end{array}\right]
$$

A é a matriz de coeficientes de parentesco entre os animais; I, uma matriz identidade. Os componentes da variância fenotípica $\left(\hat{\sigma}_{P}^{2}\right)$ estimados incluíram: $\hat{\sigma}_{a}^{2}$, variância genética aditiva direta; $\hat{\sigma}_{m}^{2}$, variância genética aditiva materna; $\hat{\sigma}_{p m}^{2}$, a variância devida aos efeitos de ambiente permanente da mãe; $\hat{\sigma}_{e}^{2}$, a variância residual, e $\hat{\sigma}_{a m}$, a covariância genética entre os efeitos direto e 
Tabela 1 - Número de observações e algumas estatísticas descritivas das características peso aos 205 (P205), aos 365 (P365) e aos 550 (P550) dias de idade

Table 1 - Number of records and some descriptive statistics of the traits weight at 205 (W205), at 365 (W365) and at 550 (W550) days of age

\begin{tabular}{lccc}
\hline Item & P205(W205) & P365 (W365) & P550 (W550) \\
\hline $\begin{array}{l}\text { Número de animais } \\
\text { Number of animals }\end{array}$ & 30.746 & 27.701 & 18.493 \\
$\begin{array}{l}\text { Número de animais em A } \\
\text { Number of animals in } A^{-1}\end{array}$ & 41.420 & 37.561 & 26.523 \\
$\begin{array}{l}\text { Média } \pm \text { desvio-padrão } \\
\text { Mean } \pm \text { standard deviation }\end{array}$ & $170,30 \pm 24,51$ & $222,40 \pm 36,29$ & $286,45 \pm 55,41$ \\
CV\% & 14,39 & 16,32 & 19,34 \\
\hline
\end{tabular}

$\mathrm{A}=$ matriz de numeradores dos coeficientes de parentesco (numerator relationship matrix).

materno. A partir das estimativas destes componentes, podem-se derivar os seguintes parâmetros: $\hat{h}_{a}^{2}=\hat{\sigma}_{a}^{2} / \hat{\sigma}_{P}^{2}$, herdabilidade direta e $\hat{h}_{m}^{2}=\hat{\sigma}_{m}^{2} / \hat{\sigma}_{P}^{2}$, herdabilidade materna.

$\mathrm{Na}$ Tabela 1 é apresentada a estrutura dos dados para as três características, assim como a média geral observada, o desvio-padrão e os respectivos coeficientes de variação.

\section{Resultados e Discussão}

As estimativas dos componentes de variância e covariância, bem como das herdabilidades e da correlação genética entre os efeitos diretos e maternos, são apresentadas na Tabela 2. As estimativas de herdabilidade aditiva direta obtidas para P205 $(0,16)$, P365 $(0,17)$ e P550 $(0,13)$ são baixas, indicando a necessidade de se utilizar informações proporcionadas por parentes no processo de seleção. As estimativas de herdabilidade aditiva materna obtidas neste estudo (Tabela 2) para P205(0,10), P365(0,03) e P550 $(0,03)$ indicam que os efeitos maternos tiveram importante influência sobre o peso à desmama, mas pouco efeito sobre os pesos pós-desmama.

As correlações genéticas entre os efeitos aditivos direto e materno foram iguais a -0,42 (P205), -0,20 (P365) e -0,28 (P550) (Tabela 2), indicando a existência de antagonismo entre esses efeitos, principalmente para P205, portanto, a seleção para melhorar os efeitos aditivos diretos deve resultar em piora nos efeitos aditivos maternos, e a seleção para qualquer um deles não deve ser efetiva.

As tendências genéticas anuais referentes aos efeitos genéticos direto, materno e do total maternal, para as características peso ao desmame (P205), ao ano (P365) e ao sobreano (P550), encontram-se na Tabela 3.

As tendências genéticas dos efeitos genéticos aditivos direto foram iguais a 0,$13 ; 0,21$ e $0,28 \mathrm{~kg} / \mathrm{ano}$, para P205, P365 e P550, respectivamente (Tabela 3). Em termos de mudança genética anual, isto representa

Tabela 2 - Componentes ${ }^{1}$ de variância e covariância, herdabilidades direta e materna ${ }^{1}$ e correlação genética entre os efeitos direto e materno ${ }^{1}$ para os pesos aos 205 (P205), 365 (P365) e 550 (P550) dias de idade

Table 2 - Variance and covariance components ${ }^{1}$, direct and maternal heritabilities ${ }^{1}$, and genetic corelation ${ }^{1}$ between the direct and maternal effects for body weights at 205 (W205), 365 (W365) and 550 (W550) days of age

\begin{tabular}{lrrrrrrrrr}
\hline Característica & $\hat{\sigma}_{P}^{2}$ & $\hat{\sigma}_{a}^{2}$ & $\hat{\sigma}_{m}^{2}$ & $\hat{\sigma}_{p m}^{2}$ & $\hat{\sigma}_{e}^{2}$ & $\hat{\sigma}_{a m}$ & $\hat{h}_{a}^{2}$ & $\hat{h}_{m}^{2}$ & $\hat{r}_{\text {Gam }}$ \\
Trait & & & & & & & & & \\
\hline P205/W205 & 427,23 & 67,24 & 42,72 & 19,02 & 320,79 & $-22,54$ & 0,16 & 0,10 & $-0,42$ \\
P365/W365 & 715,90 & 121,94 & 19,20 & 36,43 & 548,04 & $-9,71$ & 0,17 & 0,03 & $-0,20$ \\
P550/W550 & 1675,25 & 225,06 & 47,83 & 17,00 & 1414,38 & $-29,02$ & 0,13 & 0,03 & $-0,28$ \\
\hline
\end{tabular}

${ }^{1} \hat{\sigma}_{P}^{2}, \hat{\sigma}_{a}^{2}, \hat{\sigma}_{m}^{2}, \hat{\sigma}_{p m}^{2}, \hat{\sigma}_{e}^{2}, \hat{\sigma}_{a m}, \hat{h}_{a}^{2}, \hat{h}_{m}^{2}, \hat{r}_{\text {Gam }}=$ componentes de variância fenotípica, genética aditiva direta, genética aditiva materna, de ambiente permanente e residual e de covariância genética entre os efeitos aditivos direto e materno, herdabilidades direta e materna, e correlação genética entre os efeitos diretos e maternos, respectivamente.

${ }^{1} \hat{\sigma}_{P}^{2}, \hat{\sigma}_{a}^{2}, \hat{\sigma}_{m}^{2}, \hat{\sigma}_{p m}^{2}, \hat{\sigma}_{e}^{2}, \hat{\sigma}_{a m}, \hat{h}_{a}^{2}, \hat{h}_{m}^{2}, \hat{r}_{\text {Gam }}=$ phenotypic, direct additive, maternal additive, permanent environmental and residual components of variance, genetic covariance between direct and maternal effects, direct and maternal heritabities, and genetic correlation between direct and maternal effects, respectively. 
Tabela 3 - Estimativas das tendências genéticas anuais e respectivos desvios-padrão das características, para os efeitos genético direto, materno e total maternal

Table 3 - Estimates of annual genetic trends and respective standard deviations for direct, maternal and total maternal genetic effects

\begin{tabular}{lccc}
\hline & \multicolumn{3}{c}{$\begin{array}{c}\text { Média } \pm \text { desvio-padrão }(\mathrm{kg} / \mathrm{ano}) \\
\text { Mean } \pm \text { standard deviation (kg/year) }\end{array}$} \\
\cline { 2 - 4 } Características & $\begin{array}{c}\text { Efeito genético materno } \\
\text { Traits }\end{array}$ & $\begin{array}{c}\text { Maternal genetic effect } \\
\text { Deirect genetic effect }\end{array}$ & $\begin{array}{c}\text { Total maternal } \\
\text { Total maternal }\end{array}$ \\
P205(W205) & $0,1341 \pm 0,0000$ & $0,0192 \pm 0,0000$ & $0,0862 \pm 0,0000$ \\
P565 (W365) & $0,2068 \pm 0,0045$ & $-0,0107 \pm 0,0008$ & $0,0927 \pm 0,0024$ \\
P550) & $0,2758 \pm 0,0001$ & $-0,0222 \pm 0,0001$ & $0,1157 \pm 0,0001$ \\
\hline
\end{tabular}

P205, P365 e P550 = peso aos 205, 365 e 550 dias de idade, respectivamente.

$W 205, W 365$ and $W 550=$ weight at 205, 365 and 550 days of age, respectively.

incrementos de 0,$08 ; 0,09$ e $0,10 \%$ nas médias dos três pesos (Tabela 1) na ordem crescente de idade, representando uma diferença de aproximadamente $3,6 \mathrm{~kg}$ no peso à desmama, $5,6 \mathrm{~kg}$ no peso ao ano e $7,5 \mathrm{~kg}$ no peso ao sobreano, como conseqüência do progresso genético oriundo do efeito genético direto, nos 27 anos estudados. Nas Figuras 1, 2 e 3, em que são mostrados os ganhos observados e os estimados, pode-se constatar a evolução do ganho genético ao longo dos anos para P205, P365 e P550, sendo que as estimativas variaram de $-1,4$ a 2,$4 ;-2,1$ a 3,5 ; e -2,7 a 4,8 , na mesma ordem, indicando ampla variabilidade genética das características nos diferentes anos estudados.

Os progressos genéticos aditivos direto obtidos neste trabalho, apesar de significativos, estão bem aquém do que poderia ser alcançado em virtude da variação genética existente nas características. Considerando-se o período de cinco anos como o intervalo de gerações em bovinos de corte (Giannoni \& Giannoni, 1983), a intensidade de seleção (i) de 1,274 (retenção de $10 \%$ de machos e $50 \%$ de fêmeas), as herdabilidades diretas $\left(\hat{h}_{a}^{2}\right)$ da Tabela 2 , os desviospadrão fenotípicos $\left(\sigma_{\mathrm{p}}\right)$ iguais a 24,$51 ; 36,29 \mathrm{e} 55,41 \mathrm{~kg}$, seria possível obter avanços genéticos $(\Delta \mathrm{G})$ por geração $\left(\Delta \mathrm{G} /\right.$ geração $\left.=\mathrm{i} \times \hat{h}_{a}^{2} \times \mathrm{sp}\right)$ da ordem de 1,00 ; 1,57 e $1,84 \mathrm{~kg} /$ ano, para P205, P365 e P550, respectivamente, correspondendo a 0,$59 ; 0,71$ e $0,65 \%$ das médias dos pesos. Utilizando-se as herdabilidades totais $\left[\hat{h}_{T}^{2}=\left(\hat{\sigma}_{a}^{2}+0,5 \hat{\sigma}_{m}^{2}+1,5 \hat{\sigma}_{a m}\right) / \hat{\sigma}_{P}^{2}\right.$ ], e tomando os resultados da Tabela 3 , os ganhos possíveis seriam menores, em virtude do antagonismo entre os efeitos aditivos direto e materno, e seriam, na mesma ordem, iguais a 0,$94 ; 1,48$ e $1,69 \mathrm{~kg} / \mathrm{ano}$, respectivamente, correspondendo a 0,$55 ; 0,67$ e $0,59 \%$ da média dos rebanhos. Segundo Smith (1985), a taxa de mudança genética anual possível de se obter é de 1 a $3 \%$ da média da população. Desse modo, observa-se que as mudanças genéticas anuais estão abaixo do limite inferior daquele intervalo.

Incrementos, quer seja para ganho de peso até a desmama quer seja para peso à desmama ou pósdesmama, têm sido obtidos por criadores de diversas raças como atestam os resultados de Euclides Filho et al. (1986), Pimenta Filho (1986) e Silva (1990).

Por outro lado, resultados indicando baixos progressos não são raros, como os valores encontrados por vários autores em diversos rebanhos de diferentes raças, dentre os quais pode-se ressaltar Packer (1977), Eler et al. (1994), Euclides Filho et al. (1997 a, b), Silva et al. (1997).

Euclides Filho et al. (1997b) ressaltam, no entanto, que análises de tendências genéticas como esta, que são conduzidas com numerosos rebanhos distribuídos por vários estados brasileiros, e criados em vários sistemas de produção, poderiam resultar, caso as avaliações fossem realizadas em rebanhos isolados, como

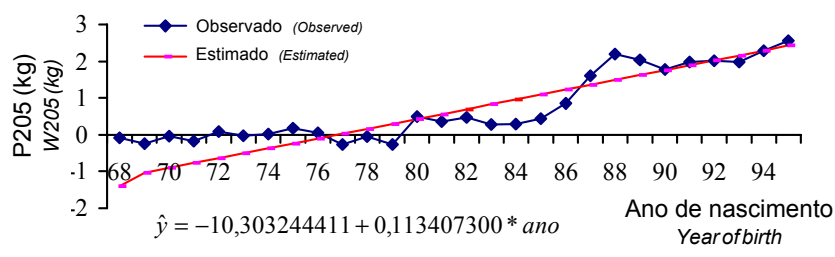

Figura 1 - Tendências genéticas $(\mathrm{kg})$ dos efeitos genéticos direto para o peso ao desmame, padronizado para 205 dias de idade (P205), no período de 1968 a 1995.

Figure 1 - Direct genetic trends $(\mathrm{kg})$ for weight at weaning standardized to 205 days of age (W205), from 1968 to 1995. 


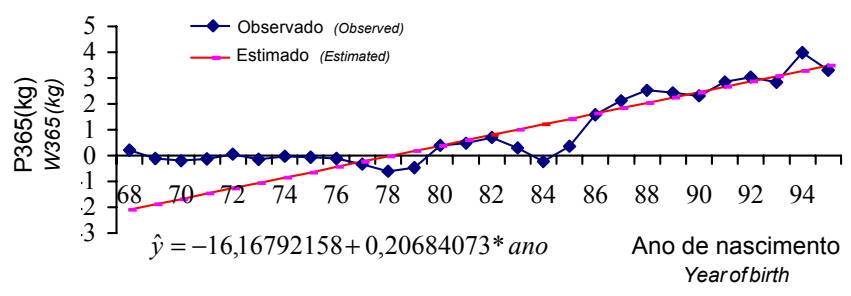

Figura 2 - Tendências genéticas $(\mathrm{kg})$ dos efeitos genéticos direto para o peso aos 12 meses, padronizado para 365 dias de idade (P365), no período de 1968 a 1995.

Figure 2 - Direct genetic trends $(\mathrm{kg})$ for weight at 12 months standardized to 365 days of age (W365), from 1968 to 1995.

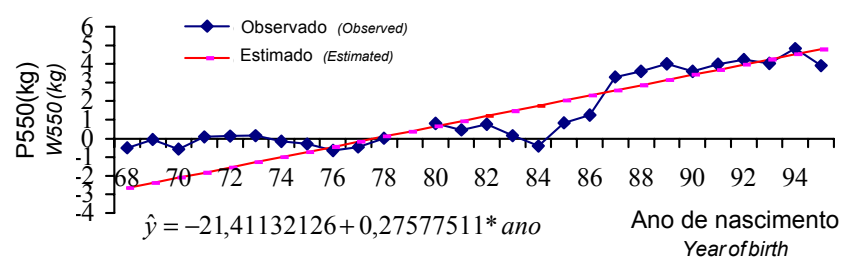

Figura 3 - Tendências genéticas $(\mathrm{kg})$ dos efeitos genéticos direto para o peso aos 18 meses, padronizado para 550 dias de idade (P550), no período de 1968 a 1995.

Figure 3 - Direct genetic trends $(\mathrm{kg})$ for weight at18 months, standardized to 550 days of age (W550), from 1968 to 1995.

fez Mello (1999), ou mesmo por região, como fizeram Ferraz Filho et al. (1997), em tendências genéticas positivas de maior valor que as verificadas no todo; podendo-se, ainda, encontrar tendências negativas, como obtiveram Souza et al. (1998).

As tendências genéticas dos efeitos genéticos maternos apresentaram-se como praticamente nulas $(0,0192 \mathrm{~kg} / \mathrm{ano})$ para P205. Resultados semelhantes foram obtidos por Nobre et al. (1988) e Euclides Filho et al. (1997 a,b).

No caso presente, a contribuição do efeito genético materno, por outro lado, foi bem menor que a do efeito direto e representou apenas $0,01 \%$ de incremento anual na média dos pesos ao desmame (Figura 4). Isto representa aumento de $0,518 \mathrm{~kg}$, aproximadamente, como resultado de contribuições do efeito genético materno, durante o período estudado,

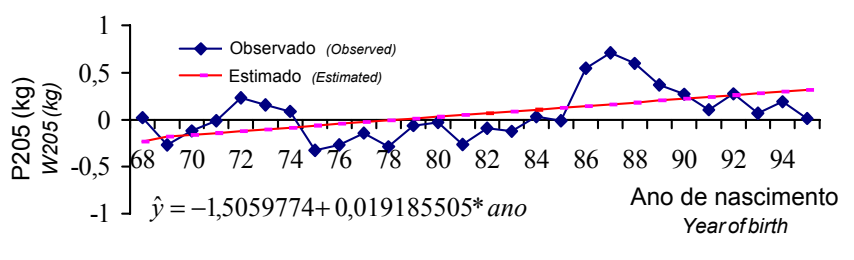

Figura 4 - Tendências genéticas $(\mathrm{kg})$ dos efeitos genéticos maternos para peso à desmama, padronizado para 205 dias (P205), no período de 1968 a 1995.

Figure 4 - Maternal genetic trends $(\mathrm{kg})$ for weight at weaning, standardized to 205 days (W205), from 1968 to 1995.

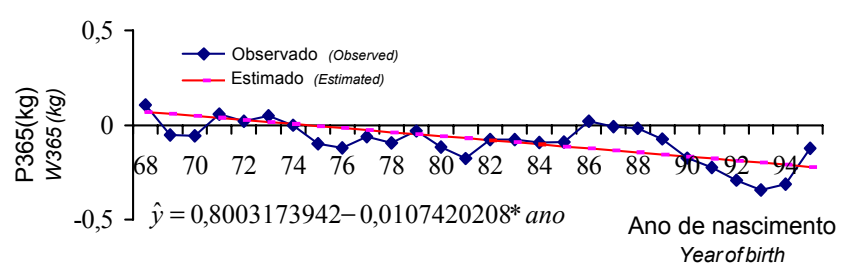

Figura 5 - Tendências genéticas $(\mathrm{kg})$ dos efeitos genéticos maternos para peso ao ano, padronizado para 365 dias (P365), no período de 1968 a 1995.

Figure 5 - Maternal genetic trends $(\mathrm{kg})$ for weight at year, standardized to 365 days (W205), from 1968 to 1995.

ou seja, $0,938 \%$ do progresso observado como conseqüência do efeito genético direto. Valores de pequena expressão foram também obtidos por Euclides Filho et al. (1986) e Silva et al. (1997), com rebanhos Nelore, e Euclides Filho et al. (1997a,b), com Indubrasil e Guzerá, em diferentes regiões brasileiras.

Verificaram-se tendências negativas para o efeito materno $(\mathrm{P}<0,001)$ para $\mathrm{P} 365(-0,01 \mathrm{~kg} / \mathrm{ano}) \mathrm{e}$ P550 (-0,02 kg/ano), representando perdas anuais de 0,005 e $0,007 \%$ em relação à media para os pesos aos 365 e 550 dias de idade, respectivamente, como observado nas Figuras 5 e 6.

As estimativas de tendência genéticas obtidas para o efeito materno nulas ou negativas devem-se à correlação negativa entre os efeitos direto e materno, aumentando-se o efeito direto (principalmente após 1980) diminui-se o materno. 


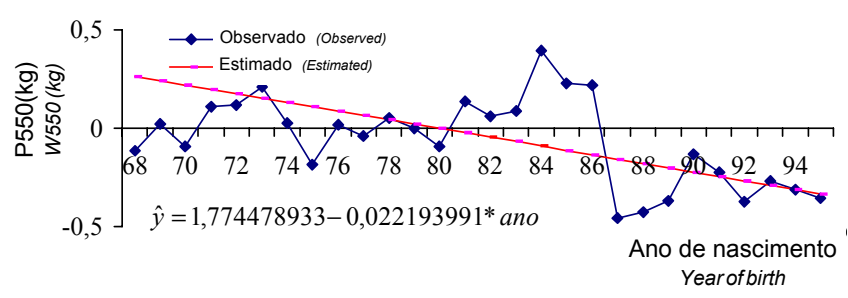

Figura 6 - Tendências genéticas $(\mathrm{kg})$ dos efeitos genéticos maternos para peso ao sobreano, padronizado para 550 dias (P550), no período de 1968 a 1995.

Figure 6 - Maternal genetic trends $(\mathrm{kg})$ for weight at yearling, standardized to 550 days (W550) from 1968 to 1995.

As tendências genéticas anuais do total maternal foram significativas $(\mathrm{P}<0,001)$ e iguais a 0,$09 ; 0,09 \mathrm{e}$ $0,12 \mathrm{~kg} /$ ano para P205, P365 e P550, respectivamente (Tabela 3), representando 0,05;0,04 e 0,04\% da média fenotípica daqueles pesos. O resultado encontrado foi bem inferior aos 0,38 e $0,40 \%$ obtidos por Mello (1999) para peso à desmama e a um ano de idade, respectivamente, em um rebanho bovino da raça Canchin.

\section{Conclusões}

Os ganhos genéticos observados para os pesos estudados foram positivos, porém bem aquém do possível, talvez em decorrência da pouca intensidade de seleção aplicada em alguns rebanhos. Técnicas modernas de avaliação genética e esforço contínuo de seleção devem ser usados por criadores e melhoristas de bovinos da raça Tabapuã para obter maiores conquistas genéticas.

\section{Literatura Citada}

BOLDMAN, K.G.; KRIESE, L.A.; Van VLECK, L.D. et al. A Manual for use of MTDFREML; a set of programs to obtain estimates of variances and covariances [DRAFT]. Lincoln: Department of Agriculture, Agricultural Research Service, 1993. 120p.

BRASIL. Ministério da Agricultura e do Abastecimento. Sumário nacional de touros das raças zebuínas: Tabapuã. edição 1999/MARA. Uberaba: Embrapa Gado de Corte/ABCZ, 1999. 39p. (Documentos/Embrapa Gado de Corte, 83)

ELER, J.P.; FERRAZ, J.B.S.; LÔBO, R.B. Estimação da tendência genética na raça Nelore, usando modelos animais univariado $\mathrm{e}$ multivariado. In: REUNIÃO ANUAL DA SOCIEDADE BRASILEIRA DE ZOOTECNIA, 31., 1994, Maringá. Anais... Maringá: Sociedade Brasileira de Zootecnia, 1994. p.171.
EUCLIDES FILHO, K.; NOBRE, P.R.C.; ROSA, A.N. Tendências genéticas em características de crescimento em gado Nelore. In: REUNIÃO ANUAL DA SOCIEDADE BRASILEIRA DE ZOOTECNIA, 23., 1986, Campo Grande. Anais... Campo Grande: Sociedade Brasileira de Zootecnia, 1986. p.311.

EUCLIDES FILHO, K.; SILVA, L.O.C.; ALVES, R.G.O. et al. Tendências genéticas na raça Indubrasil. In: REUNIÃO ANUAL DA SOCIEDADE BRASILEIRA DE ZOOTECNIA, 34., 1997, Juiz de Fora. Anais... Juiz de Fora: Sociedade Brasileira de Zootecnia, 1997a. p.171.

EUCLIDES FILHO, K.; SILVA, L.O.C.; FIGUEIREDO, G.R. Tendências genéticas na raça Guzerá. In: REUNIÃO ANUAL DA SOCIEDADE BRASILEIRA DE ZOOTECNIA, 34., 1997, Juiz de Fora. Anais... Juiz de Fora: Sociedade Brasileira de Zootecnia, 1997b. p.173.

FERRAZ FILHO, P.B.; BIANCHINI SOBRINHO, E.; SILVA, L.O.C. et al. Tendência genética em pesos de bovinos da raça Nelore Mocha no Brasil. In: REUNIÃO ANUAL DA SOCIEDADE BRASILEIRA DE ZOOTECNIA, 34., 1997. Juiz de Fora. Anais... Juiz de Fora: Sociedade Brasileira de Zootecnia, 1997. p.254-256.

GIANNONI, M.A.; GIANNONI, M.L. Genética e melhoramento de rebanho nos trópicos. São Paulo: Nobel, 1983. $463 p$.

MELLO, S.P. Tendência genética para pesos em um rebanho da raça Canchin: Jaboticabal: Faculdade de Ciências Agrária e Veterinárias, 1999. 78p. Dissertação (Mestrado em Zootecnia) - Universidade Estadual Paulista, 1999.

NOBRE, P.R.C.; ROSA, A. N.; EUCLIDES FILHO, K. Interação reprodutor $\mathrm{x}$ estação de nascimento e reprodutor $\mathrm{x}$ fazenda sobre crescimento de bezerros Nelore. Revista da Sociedade Brasileira de Zootecnia, v.17, n.2, p.120-131, 1988.

PACKER, I.U. Análise genética do crescimento até a desmama de bezerros Canchin. Piracicaba: Universidade Federal de São Paulo, 1977. 173p. Tese (livre Docência) - Escola Superior de Agricultura "Luiz de Queiroz", 1977.

PIMENTA FILHO, E.C. Mudança genética no peso aos 365 dias de idade de bovinos Nelore no Estado de São Paulo. Ribeirão Preto: Universidade Federal de São Paulo, 1986. 61p. Tese (Doutorado em Genética) - Universidade de São Paulo.

SILVA, L.O.C. Tendência Genética e Interação Genótipo $\mathbf{x}$ Ambiente em rebanhos Nelore, criados a pasto no Brasil Central. Viçosa, MG: Universidade Federal de Viçosa, 1990. 113p. Tese (Doutorado em Genética e Melhoramento) Universidade Federal de Viçosa, 1990.

SILVA, L.O.C.; EUCLIDES FILHO, K.; NOBRE, P.R.C. et al. Tendência genética na raça Nelore no Brasil. In: REUNIÃO ANUAL DA SOCIEDADE BRASILEIRA DE ZOOTECNIA, 34., 1997, Juiz de Fora. Anais... Juiz de Fora: Sociedade Brasileira de Zootecnia. 1997. p.175.

SMITH, C. Rates of genetic change in farm livestock. Research Development Agricultural, v.1, n.2, p.79-85; Animal Breeding Abstracts, v.53, n.9, p.699, 1985.

SOUZA, J.C., RAMOS, A.A., SILVA, L.O.C. et al. Tendência genética do peso ao desmame de bezerros da raça Nelore In: REUNIÃO ANUAL DA SOCIEDADE BRASILEIRA DE ZOOTECNIA, 35., 1998, Botucatu. Anais... Juiz de Fora: Sociedade Brasileira de Zootecnia, 1998. p.231.

Recebido em: 16/05/01 Aceito em: 07/11/01 